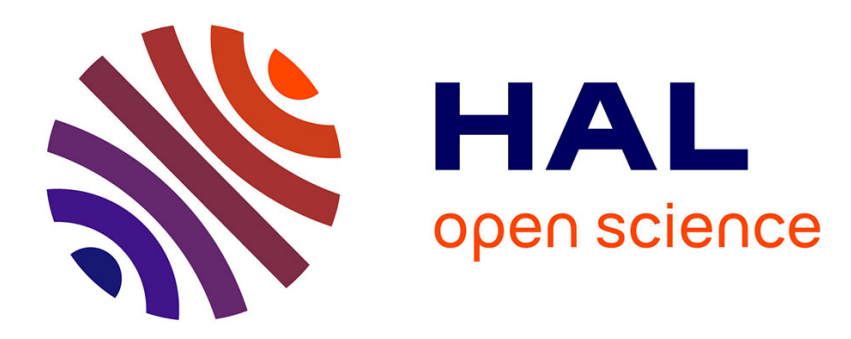

\title{
vesicles and red blood cells in shear flow
}

Manouk Abkarian, Annie Viallat

\section{To cite this version:}

Manouk Abkarian, Annie Viallat. vesicles and red blood cells in shear flow. Soft Matter, 2008, 4, pp.653. 10.1039/b716612e. hal-00321718

\section{HAL Id: hal-00321718 \\ https://hal.science/hal-00321718}

Submitted on 15 Sep 2008

HAL is a multi-disciplinary open access archive for the deposit and dissemination of scientific research documents, whether they are published or not. The documents may come from teaching and research institutions in France or abroad, or from public or private research centers.
L'archive ouverte pluridisciplinaire HAL, est destinée au dépôt et à la diffusion de documents scientifiques de niveau recherche, publiés ou non, émanant des établissements d'enseignement et de recherche français ou étrangers, des laboratoires publics ou privés. 


\title{
Vesicles and red blood cells in shear flow
}

\author{
Manouk Abkarian ${ }^{b}$ and Annie Viallat ${ }^{a, *}$ \\ Receipt/Acceptance Data [DO NOT ALTER/DELETE THIS TEXT] \\ ${ }_{5}$ Publication data [DO NOT ALTER/DELETE THIS TEXT] \\ DOI: 10.1039/b000000x [DO NOT ALTER/DELETE THIS TEXT]
}

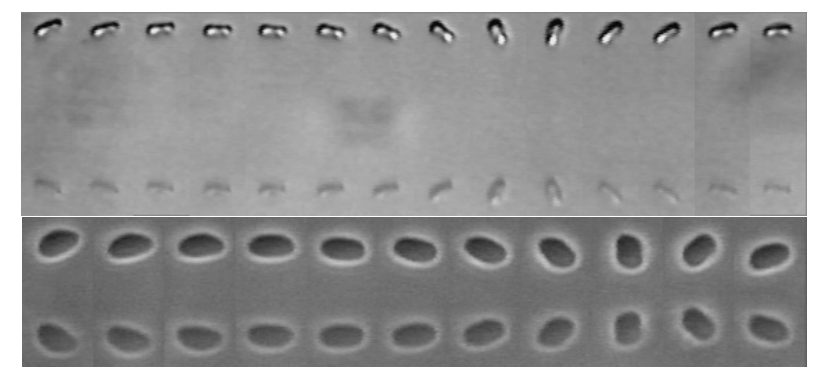

We describe the similarities and the specificities of the behaviour of individual soft particles,

10 namely, drops, lipid vesicles and red blood cells subjected to a shear flow. We highlight that their motion depends in a non trivial way on the particle mechanical properties. We detail the effect of the presence of a wall with or without wall-particle attractive interaction from a biological perspective.

\section{Introduction}

Blood circulation, microfluidic devices and emulsion processing bring into play soft micron-size particles like cells, vesicles and drops in creeping flows. We highlight the generic

20 behaviour of these particles in shear flow and its specificities induced by the differences between the various particle structural properties. We focus on three situations relevant for blood circulation: particles in infinite medium, close to a wall (blood cells at a vascular endothelium) or adhered to a wall ${ }_{25}$ (leukocytes adhered to the endothelium).

Beyond their common features, like size and fluidity, fundamental differences exist among drops, vesicles and cells: drops and surfactant-covered drops present a viscous extensible interface; while vesicle and cell membranes are

\footnotetext{
${ }^{a}$ Adhésion et Inflammation, Inserm U600, CNRS UMR 6212 Université Méditerranée, case 937, 163 av de Luminy 13288 Marseille Cedex,

France. Fax: 334918288 51; Tel: 334918288 53; E-mail: viallat@marseille.inserm.fr

${ }^{b}$ Laboratoire des Colloïdes, Verres et Nanomatériaux, CNRS UMR 5587, Université Montpellier II, Place Eugène Bataillon 34095 Cedex 05 Montpellier, France. Fax: 334671446 37; Tel: 334671435 82; Email:abkarian@lcvn.univ-montp2.fr
}

${ }_{30}$ essentially incompressible ${ }^{1}$, with no possible transport of molecules between the membrane and the inner volume, ensuring a constant membrane area. Cells exhibit more complicated mechanical properties. Red blood cells' (RBC) membrane, for instance, is underlined by a $2 \mathrm{D}$ visco-elastic 35 spectrin network $^{2}$, which confers shear elasticity to their membrane and is responsible for their biconcave discocyte shape. Numerous theoretical works have studied the dynamics of particles with different mechanical properties ${ }^{3-7}$. The approach of the problem is non trivial since the shapes are not ${ }_{40}$ given a priori and the equations coupling the the membrane tension and the surrounding flow field are nonlinear. Experimental studies have been developed by several scientific communities coming from fields as diverse as fluid mechanics, soft matter and biorheology, and therefore, were 45 not much compared to one another. Here, we describe behaviours that are common to drops, vesicles or RBCs before focussing on specific effects induced by the bending and elastic energy of the membrane and by its incompressibility.

\section{Behaviour in unbounded shear flow}


${ }_{50}$ The understanding of individual particles' behaviour in flow is a prerequisite to that of the rheology of suspensions. For instance, the deformability of RBCs plays a crucial role in the rheological properties of the whole blood, both at the largest scale of the arteries ${ }^{8}$, and in the microcirculation through very 55 small capillaries.

\section{Shapes}

A first striking feature is that drops ${ }^{9}$, vesicles ${ }^{10}$ and $\mathrm{RBCs}^{11}$ can all exhibit ellipsoidal shapes in shear flow although drop shapes are governed by surface tension, vesicle shapes are ${ }_{60}$ determined by the bending rigidity of their membrane (it is easier to bend vesicle and cell membranes than to stretch them) and RBC shapes depend on shear elasticity. These ellipsoidal shapes are, however, reached under different shear rates (small for drops and vesicles, but high for RBCs) since ${ }_{65}$ surface tension and bending elasticity involve lower energy than shear elasticity. Moreover, RBCs deform without increasing their surface area whereas, upon increasing the flow strength, the drop stretches and breaks up.

\section{Tanktreading drop-like motion in shear flow}

${ }_{70} \mathrm{~A}$ second striking point is that vesicles and RBCs characterized by a low ratio of their inner fluid viscosity to that of the suspending fluid $\left(\mathrm{c}=\eta_{\mathrm{i}} / \eta_{\mathrm{o}}\right.$ ), exhibit a typical tanktreading motion similar to that observed on drops: their membrane rotates around their centre of mass (Figure 1). This 75 rotating motion transfers the tangential stresses of the flow to the inner fluid, which rotates and dissipates the work done by external flow, allowing the particles to maintain a stationary shape and a steady stationary orientation.

\section{Transition: tumbling solid-like motion}

80 In strong contrast to drops, above a critical value of the viscosity ratio, the motion of vesicles ${ }^{10,12}$ and $\mathrm{RBCs}^{13}$ undergoes a transition towards an unsteady tumbling solidlike motion described, by analogy, with the flipping of a coin (Figure 1).

${ }_{85}$ In order to elucidate this behaviour, Keller and Skalak ${ }^{3}$ (KS) treated the particles as ellipsoids of fixed shape with a fluid membrane and used a simplified velocity field. They established the equation of evolution of the inclination angle $\theta$ of the particle with respect to the flow direction:

$\mathrm{d} \theta / \mathrm{dt}=\mathrm{A}+\mathrm{B} \cos (2 \theta)$

where $-2 \mathrm{~A}$ is equal to the wall shear rate $\gamma$, and $\mathrm{B}$ is a function of $\gamma, \mathrm{c}$ and $v$, the reduced volume defined by the ratio of the

${ }_{95}$ volume of the particle to the volume of the sphere with the same surface area. The first term on the right hand of equation (1) is a vorticity term equal to $-\gamma / 2$, while the second term is an extensional term at $45^{\circ}$ with the flow direction. Equation (1) predicts tanktreading $(-\mathrm{A} / \mathrm{B}<1)$, tumbling $(-\mathrm{A} / \mathrm{B}>1)$, and 100 a critical shear rate which depends on $\mathrm{c}$ and $v$. Theoretical predictions show a rather good qualitative agreement when compared to the observations (Figure 2) of the time evolution of $\theta$ measured on tumbling vesicles ${ }^{12}$ and $\mathrm{RBCs}^{14}$. A good quantitative agreement of the critical shear rate is also found 105 for vesicles ${ }^{12}$ (Figure 2c). However, for RBCs, the model does not capture the observed shear-rate dependency of the tanktreading-tumbling transition, and predicts shear rate at the transition in complete disagreement with observations ${ }^{15}$. The model also fails to describe the behaviour of vesicles when 110 they deform in the flow ${ }^{12}$. These discrepancies are due to the shear elasticity of the RBCs' membrane and to vesicles' deformability, we now discuss..

\section{Effect of membrane shear elasticity}

We recently showed that RBCs in shear flow present a motion 115 more complex than that described by KS. A typical behaviour $^{15}$ is illustrated in Figure 3. For high values of the shear stress $\left(\eta_{\mathrm{o}} \gamma\right)$, RBCs present a quasi steady tanktreading motion but when $\eta_{\mathrm{o}} \gamma$ is decreased their inclination oscillates about a mean angle down to a critical $\eta_{\mathrm{o}} \gamma_{\mathrm{c}}$, for which RBCs 120 tumble at least once. This swinging regime is characterized by a quasi-nondeformed cell shape and an oscillation period of the inclination angle equal to half the tanktreading period. The swinging/tumbling transition is induced by tuning down (or up) $\gamma$.

125 Swinging and shear-stress triggered transition are not observed on purely viscous vesicles but swinging has, however, been detected on elastic capsules in shear flow ${ }^{16}$, when they were slightly non spherical at rest. These features are a signature of the membrane shear elasticity. Indeed, if 130 one assumes that the unstrained RBC shape is biconcave, the local elements of the elastic membrane are not equivalent: for instance, during tanktreading, the elements which form the rim at rest rotate about the stationary cell shape to reach the dimples after rotation and vice and versa. They are then 135 locally strained and store elastic energy (Figure 4). After a $180^{\circ}$ rotation, the elements retrieve their initial shape and are no more strained, thus resulting in a periodic storage of energy. We described this effect by extending the KS model ${ }^{15}$ : when writing that the rate of dissipation of energy in the cell 140 equals the rate at which work is done by the external fluid on the cell, we added the elastic power stored in the periodic elastic membrane strain to the cell viscous energy dissipation. The modified model indeed predicts time oscillations (figure 5), and it captures the trends of the $\gamma$ variations of the 145 amplitude and period of oscillations, as well as the motion transition for decreasing values of the shear stress (Figure 3 ). We emphasize that the fit by such a model of the characteristics of swinging and tumbling-tanktreading transition on individual flowing RBCs allows the 150 determination of internal viscosity, membrane viscosity and shear modulus of each cell. Therefore, this analytical model, which still has to be refined, or alternatively, a numerical approach $^{17}$ hold promises for applications in non-invasive cellular-scale diagnostic in clinical hemorheology..

\section{${ }_{155}$ Effect of vesicle deformability}

Vesicles are floppy objects whose shape may vary during motion in the flow. In the tumbling regime, they may tremble or successively present ellipsoidal and spherical shapes, which strongly affect the flipping motion ${ }^{12,18}$. This effect, although 160 not relevant for cells which are more rigid, has received recent attention $^{5}$. It can be understood by considering that the variation of the tumbling angle with time induces a variation 
of the stretching ratio of the deformed vesicle since hydrodynamic pressure induces stretching along the $+45^{\circ}$ ${ }_{165}$ direction and compression along the $-45^{\circ}$ direction, where interior flow relaxation can lead to nearly vanishing membrane tension (in case of low internal viscosity) and hence strong shape fluctuations and/or shape relaxation into a sphere.

\section{${ }_{170}$ Presence of a wall: lateral drift}

The presence of a wall is a situation most relevant to blood physiology, especially for blood cells circulating in arterioles or venules. Indeed, a RBC depleted region near the capillary walls has been long-reported ${ }^{11,19}$ originating from the 175 impossibility for RBCs to approach the endothelium walls, while oppositely, white blood cells can approach the endothelium and be captured onto its surface.

Migration from the wall is known for deformable drops ${ }^{20}$ and $\mathrm{RBCs}^{11,19}$ since the 1960 s and has been recently observed 180 on giant lipid vesicles ${ }^{21,22}$ (figure 6). It has been explained in terms of modifications of the flow field induced by the asymmetric shape of the object and the wall ${ }^{23,24}$. The drift velocity away from the surface in absence of external force has been theoretically predicted ${ }^{24}$. No lift force is observed on 185 spherical objects.

The associated lift force $\mathrm{Fl}$ is a biological issue since it may counterbalance the adhesion force experienced by leukocytes at their approach to the endothelium. We used giant unilamellar vesicles to determine the variation of the lift force 190 with the aspect ratio, the vesicle radius, the distance to the substrate and the shear rate ${ }^{21}$. These vesicles (closed lipid bilayer membrane) were filled with sucrose solutions of controlled density ${ }^{25}$, which allowed to vary $v$ by deflating/inflating the vesicles via regulating the outer 195 osmotic pressure. Flowing vesicles were found to unbind from the substrate above a critical value of $\gamma$ and hover at a distance $\mathrm{h}$ from the substrate: $\mathrm{h}$ self-adjusts so that the hydrodynamic lift exactly counterbalances the vesicle buoyancy. In our experiments, the lift force F1 ranged from 0.2 to $150 \mathrm{pN}$ and 200 wrote as:

$$
\mathrm{Fl}=\mathrm{f}(v) \eta_{\mathrm{o}} \gamma \mathrm{R}^{3} / \mathrm{h}
$$

where $f$ was determined experimentally.

${ }_{205}$ By using Eq. (2), we estimated the lift force acting blood cells in post-capillary venules, where the wall shear stresses range from 0.2 to $1 \mathrm{~Pa}$. For $\mathrm{h}=350 \mathrm{~nm}$ (typical size of leukocytes microvilli $\left.{ }^{26}\right), \mathrm{F} 1$ ranges from 31 to $155 \mathrm{pN}$ for $\mathrm{RBCs}(v=0.7)$. It is equal to 0 for spherical leukocytes while it ranges from 21046 to $230 \mathrm{pN}$ if the cell is slightly deformed $(v=0.95)$. Hydrodynamically speaking, flowing leukocytes must therefore imperatively preserve their spherical shape to approach vessel walls. Then, selectins binders fast form and break molecular bridges with the cell, preventing its

215 deformation, allowing its capture and slowing down and permitting progressive elimination of the lubrication layer at the vessel surface. When an intimate cell-wall contact occurs, the cell no longer experiences the lift force. It can strongly bind to the endothelium and deform. Particles adhered to the 220 wall

\section{Particles adhered to the wall}

When a soft particle is strongly adhered to the wall in shear flow a new question arises: does the particle present a surface/volume flow? Or, in biological terms: are cell 225 membranar adhesion receptors able to flow towards the cell adhesion line with the substrate and enhance cell adhesion?

We experimentally showed the existence of a surface flow on giant lipid vesicles ${ }^{28}$. It is divided into two symmetric quadrants with two stagnation points on each vesicle side 230 (Figure 7). The surface streamlines avoid the motionless contact zone thus limiting the friction between the cell and the substrate. As a consequence, the membrane is strongly sheared since the rotational velocity on streamlines close to the contact zone is much smaller than that close to the 235 stagnation points.

For cells, the cytoskeleton prevents the surface flow of micron-size defects ${ }^{29}$. It could, however, allow molecular surface flow among the membrane proteins anchoring the underlying cytoskeleton and thus favouring the recruitment in 240 the contact zone of the adhesion receptors of the cell membrane. This point has still to be investigated.

\section{Conclusions}

The behaviour of soft particles in flow present specific features which strongly and non-trivially depend on the ${ }_{245}$ particles' shape and mechanical properties. These specificities must be accounted for when one considers cells in a flow, since they can be exploited to characterize individual cell mechanics (swinging), and because they involve relevant forces or flows. A lot of work has still to be done in the 250 direction of more complex biomimetic vesicles ${ }^{30}$ and cells (for instance tumor cells at the origin of metastasis), towards the effect of interactions between cells (high cell concentration in the blood flow) or to study flows that mimic blood microcirculation through micron-size capillary networks ${ }^{31}$.

\section{${ }_{255}$ Notes and references}

1 Structure and dynamics of membranes, Handbook of biological physics, ed. R. Lipowsky and E. Sackmann Elsevier, Noth Holland, 1995

2 N. Mohandas and E. Evans, Annu. Rev. Biophys. Biomol. Struct., $260 \quad 1994,23,787$

3 Keller, S., and R. Skalak, J. Fluid Mech., 1982, 120, 27-47

4 J. M. Rallison, Annu. Rev. Fluid Mech., 1984 16, 45

5 M. Kraus, W. Wintz, U. Seifert, and R. Lipowsky, Phys. Rev. Lett., 1996, 77, 3685 ; U. Seifert, Eur. Phys. J. B, 1999, 8, 405 ; H. Noguchi, and G. Gompper, Phys. Rev. Lett., 2004, 93, 8102; J. Beaucourt, F. Rioual, T. Seon, T. Biben and C. Misbah, Phys. Rev. E, 2004, 69, 011906; P. M. Vlahovska and R. S. Gracia, Phys. Rev. E, 2007, 75, 016313

6 T.W. Secomb, and R. Skalak, Q. J. Mech. Appl. Math., 1982, XXXV 2, 233

7 P. Olla, Physica A, 2000, 278 , 87; D. Barthes-Biesel and H. Sgaier, Physica A, 1991, 172, 103 ; S. Ramanujan and C. Pozrikidis, J. Fluid Mech, 1998, 361, 117 ; C. D. Eggleton and A. S. Popel, Phys. Fluids, 1998, 10, 1834-1845

2758 S. Chien, Ann. Rev. Phys., 1987, 49, 177 
9 R. G. Cox, J. Fluid Mech., 1969, 37, 601

10 de Haas, K. H., C. Blom, D. van den Ende, M. G. H. Duits, and J. Mellema, Phys. Rev. E, 1997, 56, 7132-7137

11 T. Fischer and H. Schmid-Schnbein, Blood Cells, 1977, 3, 351; T. Fischer, M. Stöhr-Liesen, and H. Schmid-Schönbein, 1978, Science, 202, 894 ; R. Tran-Son-Tay, S. Sutera, and P. Rao, Biophys. J., 1984, 46, 65 ; P. Gaehtgens and H. Schmid-Schônbein, Naturwissenschaften, 1982, 69, 294-296

12 M. -A. Mader, V. Vitkova, M. Abkarian, A. Viallat and T. 285 Podgorski, Eur. Phys. J. E, 2006, 19, 389 ; V. Kanstler and V. Steinberg, Phys. Rev. Lett., 2005, 95, 25801

13 H. Goldsmith and J. Marlow, Proc. R. Soc. Lond. B., 1972, 182, 351

14 M. Abkarian, Ph.D. Thesis, University of Grenoble, France, 2002

15 M. Abkarian, M. Faivre and A. Viallat, Phys. Rev. Lett., 2007,98 , 188302

16 K. Chang andW. Olbricht, J. Fluid Mech., 1993, 250, 609 (1993); A. Walter, H. Rehage, and H. Leonhard, Colloids Surf. A, 2001, 183, $123(2001)$

17 S. Kessler, R. Finken, and U. Seifert, arxiv :0709.2610v1 (condmat.soft) 17 Sep 2007

18 V. Kantsler and V. Steinberg, Phys. Rev. Lett., 2006, 96, 036001

19 H. L. Goldsmith, G. R. Cokelet, and P. Gaehtgens, Am. J. Physiol., 1989, 257, H1005 ; T. W. Secomb in Modeling and simulation of Capsules and Biological Cells, ed. C. Pozrikidis, Chapman \&

300 Hall/CRC mathematical biology and medecine series, London, 2003, 163

20 Chaffey, C. E., H. Brenner, and S. G. Mason, Rheol. Acta., 1965, 4, 64

21 M. Abkarian and A. Viallat, Biophys. J., 2005, 89, 1055; M.

305 Abkarian, C. Lartigue, and A. Viallat, Phys. Rev. Lett., 2002, 88, 8103-8107

22 B. Lorz, R. Simson, J. Nardi, and E. Sackmann, Europhys. Lett., 2000, 51, 468-474; Razpet, A., G. Gomis `č , V. Arrigler, S. Svetina, and B. Z` eks ${ }^{\sim}$.Eur. J. Physiol. 2000, 439(Suppl), R141-R142.

31023 U. Seifert, Phys. Rev. Lett., 1999, 83, 876-879 ; I Cantat and C. Misbah, Phys. Rev. Lett., 83, 880-884 ; S. Sukumaran and U. Seifert, Phys. Rev. E, 2001, E. 64, 1-11

24 P. Olla, Journal of Phys.ics A, 1997, 30, 317-329 ; P. Olla, Journal de Physique II, 1997, 7, 1533-1540 ; P. Olla, Phys. Rev. Lett., 1999, 82, $453-456$

25 M. Abkarian, C. Lartigue and A. Viallat, Phys. Rev. E, 2001, 63, 1-7

26 J. O. Shao, H. P. Ting-Beall, and R. M. Hochmuth, Proc. Natl. Acad. Sci. USA, 1998, 95, 6797-6802

27 C. Pozrikidis, personal communication

32028 V. Vezy, G. Massiera and A. Viallat, Soft Matter, 2007, 3, 844-851

29 Viallat, M. Faivre, M. Abkarian, C. Vézy and N. Glade, presented in part at the Gordon Research Conference colloidal, macromolec and polyelect solutions, Ventura, USA, February, 2004

30 Viallat, J. Dalous and M. Abkarian, Biophys. J., 2004, 86, 2179-2187;

325 E. Helfer, S. Harlepp, L. Bourdieu, J. Robert, F.C. MacKintosh and D. Chatenay, Phys. Rev. E, 2001, 63, 021904; H. Ringsdorf, E. Sackmann, J. Simon and F. M. Winnik, Bioch. Biophys. A., 1993, 1153, 335-344; O. Stauch, T. Uhlmann, M. Fröhlich, R. Thomann, M. El-Badry, Y/-K. Kim and R. Schubert, Biomacromolecules, 2002, 3,

330 324-333; O. Stauch, R. Schubert, G. Savin and W. Burchard, Biomacromolecules, 2004, 3, 565-578; A. Jesorka, M. Markström and O. Orwar, Langmuir, 2005, 21, 1230-1237; M. Markström, A. Gunnarsson, O. Orwar and A. Jesorka, Soft Matter, 2007, 3, 587 - 595

31 S. Shevkoplyas, T. Yoshida, S. C. Gifford and M.W. Bitensky, Lab Chip, 2006, 6, 914-920 


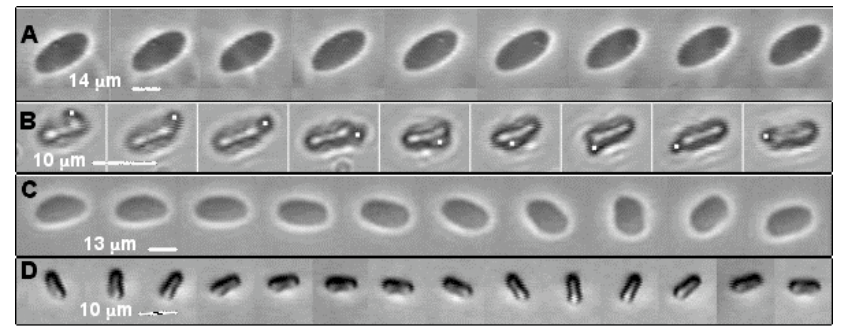

Figure 1 : Vesicles and red blood cells in shear flow. A : tanktreading vesicle, viscosity ratio $\mathrm{c}=\eta_{\mathrm{i}} / \eta_{\mathrm{o}}=1$; B : rotation of a bead (diameter $1 \mu \mathrm{m}$ ) stuck on the membrane of a tanktreading $\mathrm{RBC}$ with $\mathrm{c}=1 / 47$, shear rate $: 6 \mathrm{~s}^{-1} ; \mathrm{C}:$ tumbling vesicle with $\mathrm{c}=8$; ${ }_{340} \mathrm{D}$ : tumbling RBC with $\mathrm{c}=1 / 47$, shear rate $: 0.8 \mathrm{~s}^{-1}$
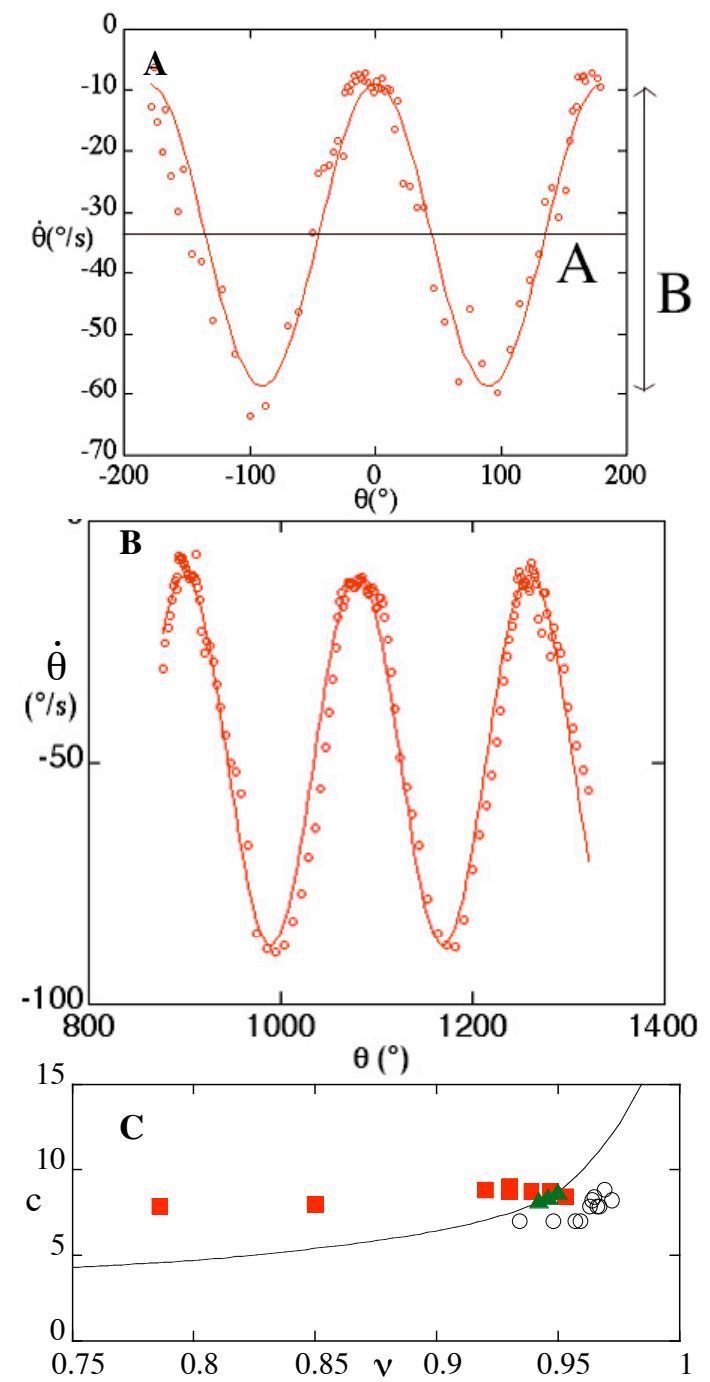

Figure 2 : Tumbling of vesicles and RBCs. Variation of the angular velocity $\mathrm{d} \theta / \mathrm{dt}$ of the inclination of a vesicle (A) and of a RBC (B) versus the inclination angle $\theta$; $\mathrm{C}$ : regimes of motion of vesicle $(\boldsymbol{\square})$ : tumbling, $(\mathrm{O})$ : tanktreading, $(\boldsymbol{\Delta})$ : transition. The solid line is the tumbling/tanktreading transition line given by the KS model. 

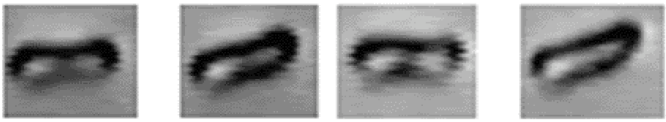

\section{$10 \mathrm{~s} \quad 20 \mathrm{~s} \quad 30 \mathrm{~s} \quad 40 \mathrm{~s}$}
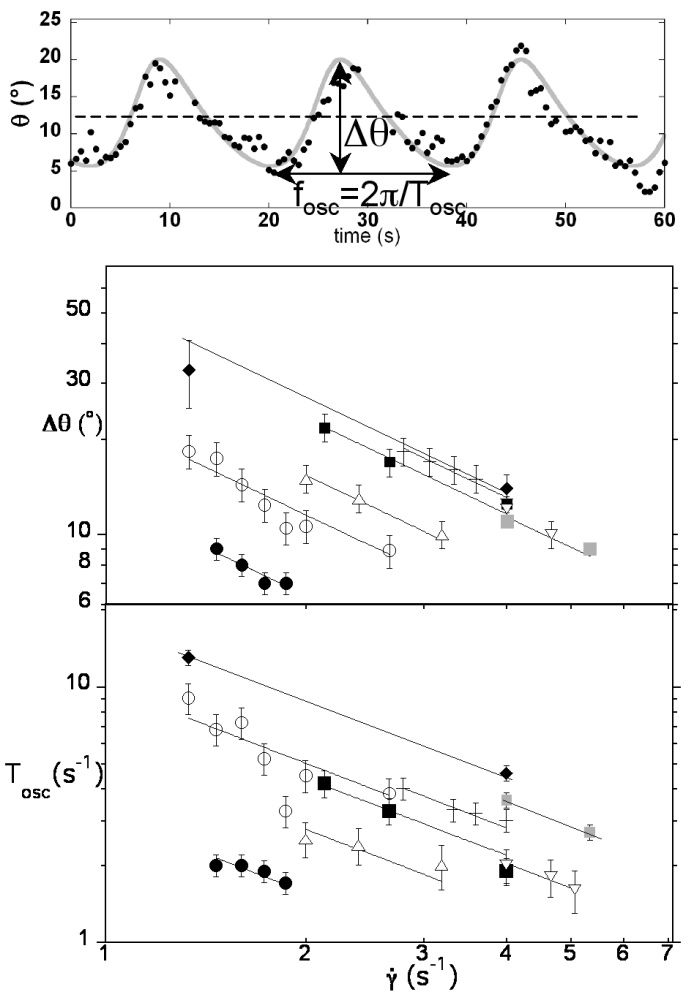

Figure 3 : RBC motion in shear flow. A : swinging $\mathrm{RBC}, \mathrm{c}=1 / 47$, shear rate $=1.33 \mathrm{~s}^{-1} ; \mathrm{B}$ : Orientation versus the time ( $\mathrm{c}=1 / 47$, shear rate $\left.=0.8 \mathrm{~s}^{-1}\right) ; \mathrm{C}$ : Experimental variations of the amplitude and period of oscillations on individual $\mathrm{RBCs},(\mathrm{O}),(\mathbf{O})$ : $\mathrm{c}=1 / 22 ;(\mathbf{\square}),(\quad): \mathrm{c}=1 / 31 ;(\Delta),(+),(\bullet),(\nabla): \mathrm{c}=1 / 47$. Solid lines are obtained by KS modified model.
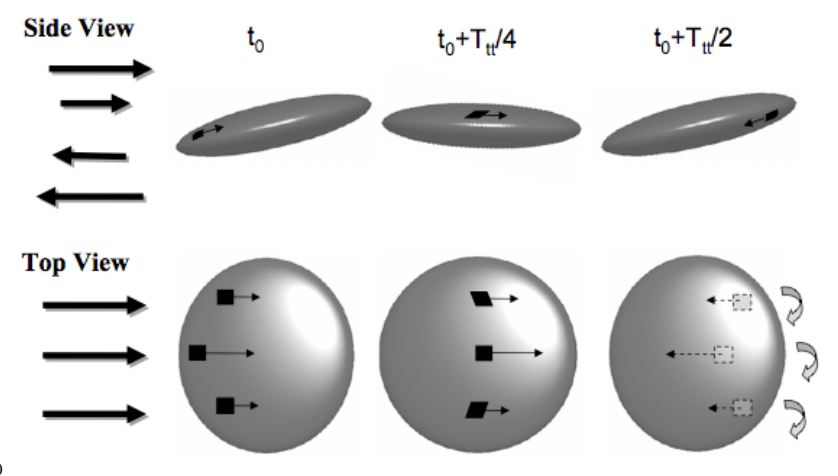

Figure 4: local strain of elastic RBC membrane elements during the rotation of the membrane when the RBC tanktreads 


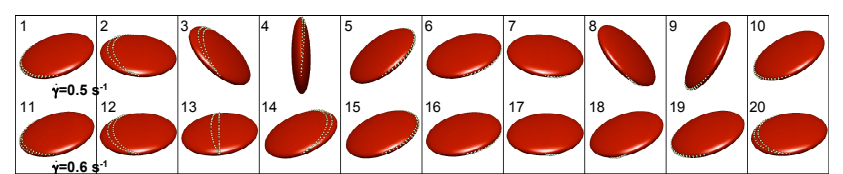

Figure 5: RBC motion and transition for two shear rates, as computed by using the modified KS model
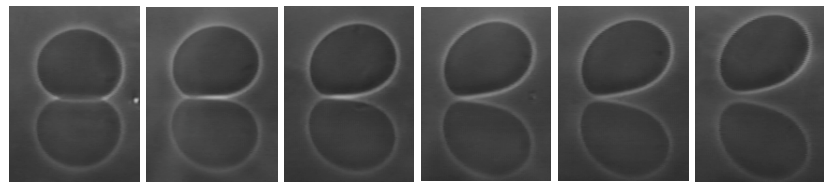

Figure 6: Unbinding of a vesicle in a shear flow. The lowest image is the reflection on the substrate. From left to right, each picture is taken at increasing shear rates: $0,0.09,0.14,0.23,0.32,0.36 \mathrm{~s}^{-1}, \mathrm{R}=36,9 \mu \mathrm{m}, v=0.98$
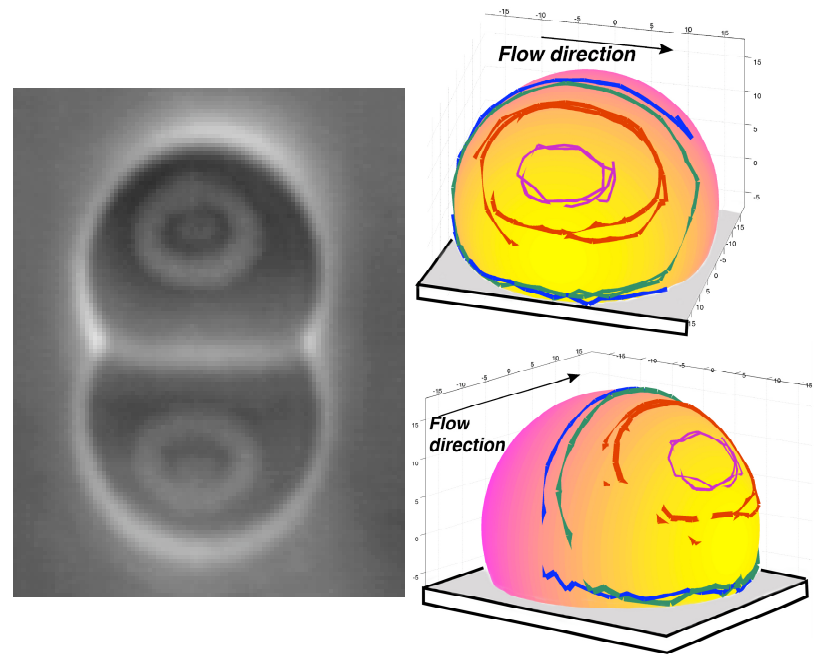

Figure 7: Marker trajectories on the surface of a vesicle adhered to a substrate in a shear flow. Left : vesicle $(\mathrm{R}=10 \mu \mathrm{m})$, its reflection on the substrate and the trajectories of two markers. One is on at stagnation point, the other one rotates on the membrane. Right : streamline reconstruction on a vesicle $(\mathrm{R}=17.5 \mu \mathrm{m})$ 